\title{
Erweiterung des genetischen Codes
}

\section{Pyrrolysyl-tRNA-Synthetase: Methano- genese und Gencode-Erweiterung}

NIKOLAJ GEORG KOCH ${ }^{1,2}$, NEDILJKO BUDISA ${ }^{1,3}$

${ }^{1}$ INSTITUT FÜR CHEMIE, TU BERLIN

2 INSTITUT FÜR BIOTECHNOLOGIE-BIOANALYTIK, TU BERLIN

${ }^{3}$ DEPARTMENT OF CHEMISTRY, UNIVERSITY OF MANITOBA, WINNIPEG, KANADA

\section{Pyrrolysyl-tRNA synthetase (PyIRS) is an enzyme of some methano-} genic Archaea for the natural incorporation of pyrrolysine into proteins. The discovery of PyIRS as a natural tool for genetic code expansion paved the way for site-specific incorporation of non-canonical amino acids (ncAAs) into proteins, with versatile side chains useful in biotechnology. Almost 20 years after the discovery, we describe the journey which led to three distinct classes of PyIRSs with unique ncAA recognitions.

DOI: $10.1007 / \mathrm{s} 12268-021-1653-\mathrm{X}$

(C) Die Autoren 2021

Die Grundlage allen Lebens sind Proteine. Sie katalysieren alle essenziellen Reaktionen in Organismen, ohne die Leben auf der Erde unmöglich wäre. Fast alle Lebewesen verwenden dafür dieselben $20 \alpha$-Aminosäuren (kanonische) als Bausteine. Der Bauplan für Proteine ist durch die DNA- und deren korrespondierende RNA-Sequenz codiert (Abb. 1). Spezifisch besteht die Information für eine Aminosäure aus einem Basentriplet, welches ein Codon bildet. Es gibt 64 solcher Codons. 61 Codons spezifizieren, welche Aminosäure an welcher Stelle einer RNASequenz eingebaut wird, und drei Codons terminieren die Proteinsequenz (StoppCodons). Da alle Lebewesen die gleichen 20 Aminosäuren für die Synthese aller Proteine verwenden, ist ein gezieltes Manipulieren einer spezifischen Aminosäure in einer Proteinsequenz, z. B. durch chemische Reaktionen, fast unmöglich.

Ein großes Ziel war es deshalb, andere Aminosäuren durch die Erweiterung des genetischen Codes ortsspezifisch einzuführen, um Proteine zur Funktionsaufklärung gezielt manipulieren oder ihre Funktionen erweitern zu können [1]. Eine Methode, um das zu bewerkstelligen, ist das gezielte Umprogrammieren eines der drei StoppCodons. Hierzu werden in einen Organismus tRNA und die dazugehörige AminoacyltRNA-Synthetase eingeführt, welche nicht mit der endogenen Translationsmaschinerie

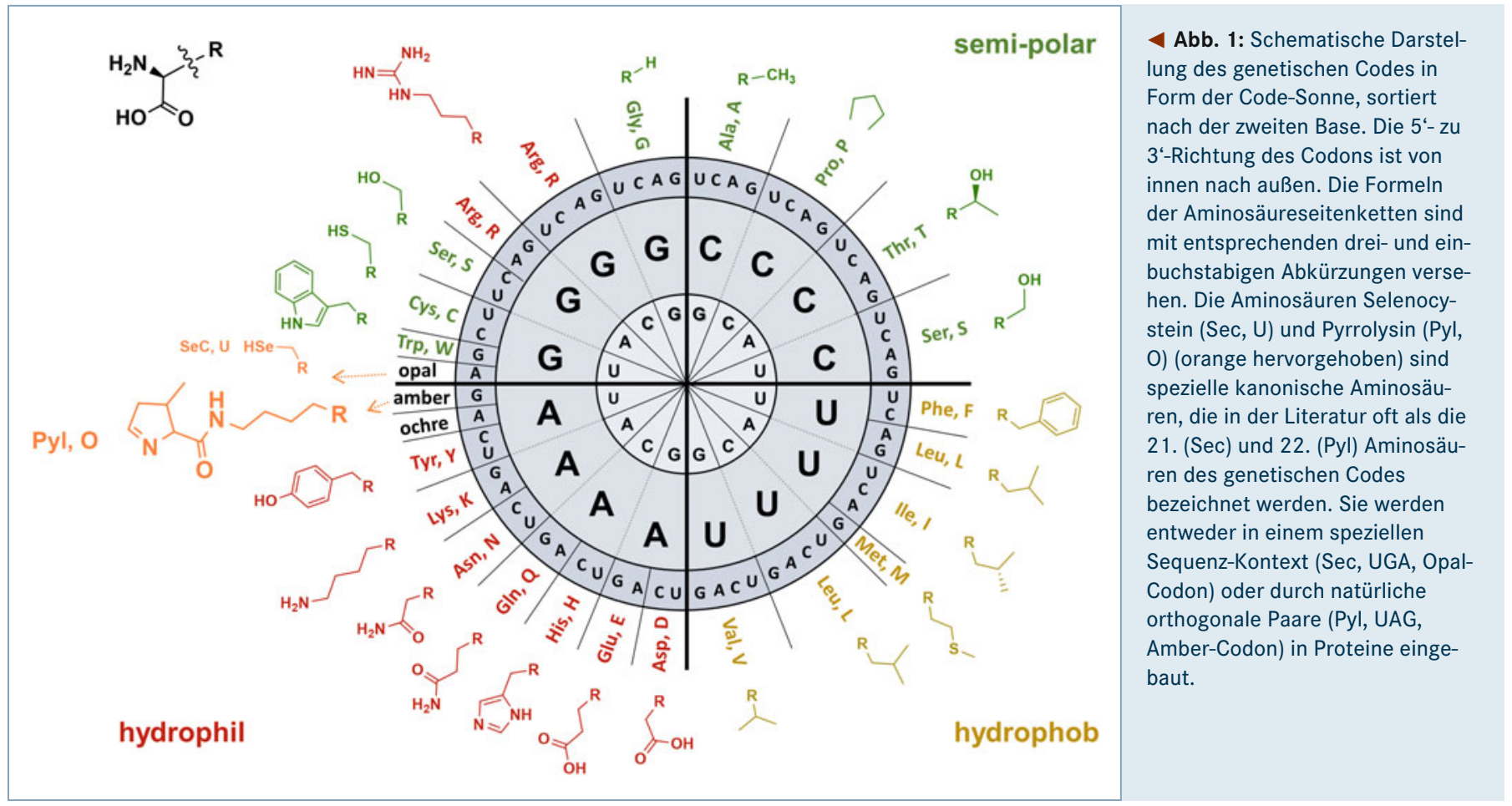


interagiert (also orthogonal ist) und außerdem so designt wurden, dass sie ausschließlich eine nicht kanonische Aminosäure (nkAS) in Korrespondenz zu einem StoppCodon in die Proteinsequenz einführen. Erstmalig gelang das in Escherichia coli mit einem orthogonalen Phenylalanin-System aus der Hefe [2]. Später wurden auch verbesserte orthogonale Translationssysteme (OTS) aus Archaeen entwickelt [3]. $\mathrm{Zu}$ den von Menschen entwickelten Systemen kam durch eine serendipitäre Entdeckung ein natürlich vorkommendes hinzu. Bei der Aufklärung der Methanogenese eines speziellen Methanbildners, Methanosarcina barkeri, wurde in dem Enzym MonomethylaminMethyltransferase ein Amber-Stopp-Codon in der DNA-Sequenz gefunden. Durch die Röntgenstrukturaufklärung dieses Enzyms wurde herausgefunden, dass das Amber-StoppCodon für eine weitere kanonische Aminosäure codiert (die 22., nach Selenocystein) [4]. Es handelte sich um ein Lysin-Derivat mit einem 4-Methyl-Pyrroline-5-CarboxylatRing verknüpft am $\mathrm{N}^{\varepsilon}$ des Lysins, welches auf den Namen Pyrrolysin (Pyl) getauft wurde (Abb. 1).

\section{Verflechtung der Evolutions- geschichte von Pyrrolysin mit „Alien“- Leben}

Höchstwahrscheinlich sind wir Zeugen eines evolutionären Prozesses der Umwidmung des Amber-Stopp-Codons der MethanosarcinaArten, welche in Lebensräumen gedeihen können, die für andere Organismen unwirtlich sind. Diese Umgebungen (z. B. Rinderpansen oder die tiefe Biosphäre des Meers) werden zwar auch von anaeroben Bakterien bewohnt, aber von Archaeen dominiert. Archaeen gehören einer Lebensdomäne an, die sich phylogenetisch sowohl von Eukaryoten als auch von Bakterien deutlich unterscheidet [5].

Es wird auch spekuliert, dass extraterrestrisch bewohnbare Zonen und Planeten mit Methanvorkommen in der Atmosphäre außerirdische Lebensformen beherbergen könnten, die methanogenen Archaea-Zellen ähnlich oder mit ihnen verwandt sind [6]. Da die Methanogenese ein einzigartiger Stoffwechsel ist, führt sie in diesem Zusammenhang zur Umcodierung von Organismen, indem Pyrrolysin als spezielle kanonische Aminosäure in ihren genetischen Code aufgenommen wird. Dies ist nicht überraschend, denn jüngste phylogenetische Analysen weisen eindeutig darauf hin, dass

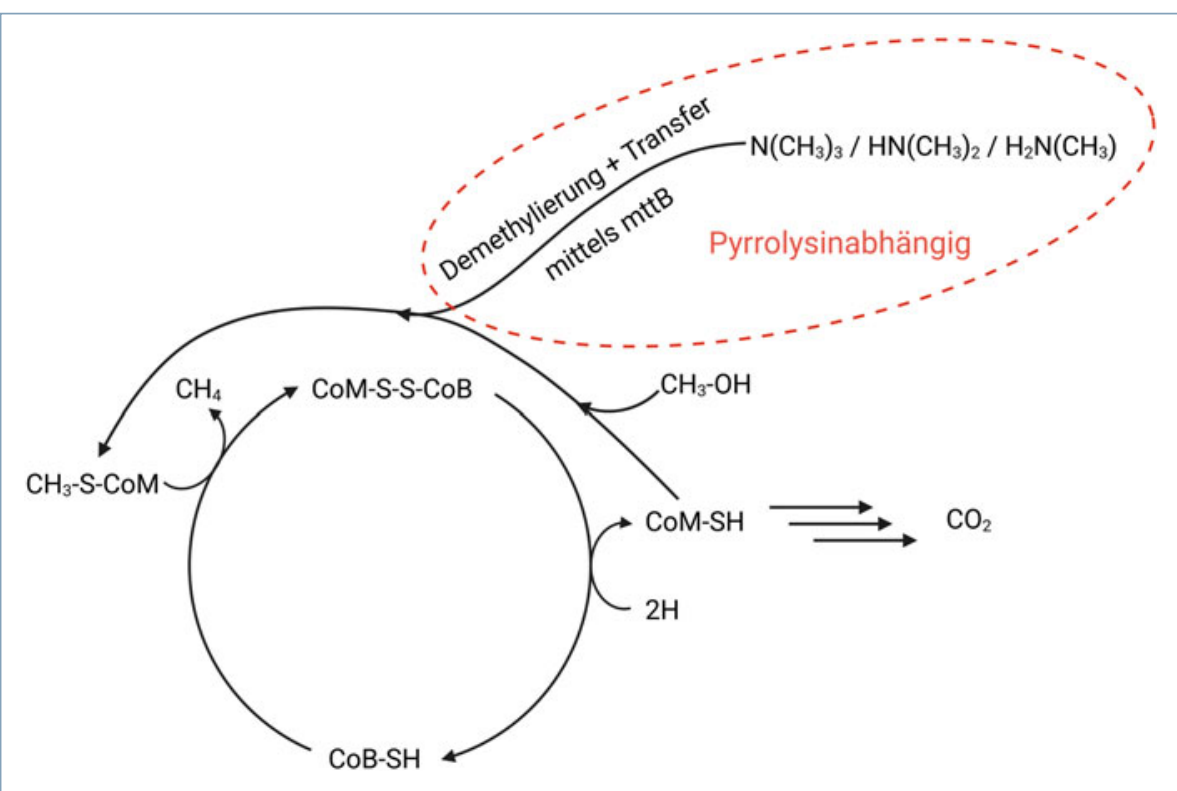

$\Delta$ Abb. 2: Schematischer Mechanismus der methylotrophen Methanogenese aus Methanol am Beispiel von Trimethylamin (TMA). Zu beachten ist, dass die Seitenkette der speziellen kanonischen Aminosäuren Pyl für den Demethylierungsprozess unerlässlich ist. Diese evolutionäre Neuerung erforderte eine Erweiterung des genetischen Codes in Methanosarcinae und ermöglichte so einen ungewöhnlichen Stoffwechsel in extremen Lebensräumen. CoM-SH: Coenzym-M-Thiol; CoB-SH: Coenzym-B-Thiol; CoM-S-S-CoB: Coenzym-M-Coenzym-B-Heterodisulfid; mttB: TMA-Methyltransferase-Gen. Mit BioRender.com erstellt.

PylRS ein evolutionäres Überbleibsel einer frühen archaealen Innovation des genetischen Codes ist [7].

Kürzlich haben wir das Evolutionsmodell „Alanin-Welt“ vorgeschlagen, das erklärt, wie der alte genetische Code (in der RNAWelt) mit nur vier Aminosäurebausteinen begann: Alanin (Ala), Glycin, Prolin (Pro) und Arginin/Lysin-Äquivalente (wie Ornithin oder Pyl). Die für den Stoffwechsel und die Translation ausgewählten Aminosäuren sind hauptsächlich Derivate von Ala, was durch die erdspezifische geochemische Umgebung in der Evolution des Lebens diktiert wurde. Es kann darüber spekuliert werden, ob auf der Erde oder in einem anderen Teil des Universums, bei Wiederholung der Geschichte, ein auf Pyrrolysin basierendes Leben hätte entstehen können, das eine grundlegend andere Chemie (Stoffwechsel) und/oder sogar eine andere Energetik des Lebens aufweist [8].

Nichtsdestotrotz haben wir jetzt eine gute Möglichkeit, diese Optionen aus der evolutionären Vergangenheit wieder aufzugreifen und experimentell parallele biologische Welten zu schaffen, wie z. B. eine potenzielle Pyrrolysin-Welt. Es sei auch darauf hingewiesen, dass Pyrrolysin als ein zusammengesetztes Molekül betrachtet werden kann, das aus methyliertem/oxidiertem Prolin-
Analogon und Lysin besteht (siehe Pyl, Abb. 1). Kürzlich wurde eine Reihe von PylDerivaten mit D-Prolin und Analoga erzeugt und in Proteine eingebaut, die ihnen einzigartige katalytische Eigenschaften verleihen (Dieter Söll, persönliche Kommunikation). Sowohl Pro als auch Pyl sind nicht nur die ältesten Aminosäuren im Aminosäurerepertoire des genetischen Codes, sondern auch wichtig für die Produktion von homochiralen Zuckern und die Vermittlung von chemischen Transformationen, die in den frühen Stadien der Evolution lebenswichtig waren (wie Transfer-Hydrierungen oder Transaminierungen) [9].

\section{Entdeckung des natürlichen Pyrrolysyl-tRNA-Synthetase-Systems}

Pyrrolysin spielt eine essenzielle Rolle in der Methanogenese aus Methylaminen. Generell gibt es drei Arten der Methanogenese: (i) Die am weitesten verbreiteten Variante verwendet $\mathrm{H}_{2}$ und $\mathrm{CO}_{2}$, um Methan zu synthetisieren. (ii) Die Bildung aus Acetat. (iii) Die am wenigsten verbreitete Variante, die methylotrophe Methanogenese aus organischen Substraten, die eine Methylgruppe beinhalten. Sie wird hauptsächlich von Organismen aus der Ordnung der Methanosarcinales durchgeführt (Abb. 2, [10]). 


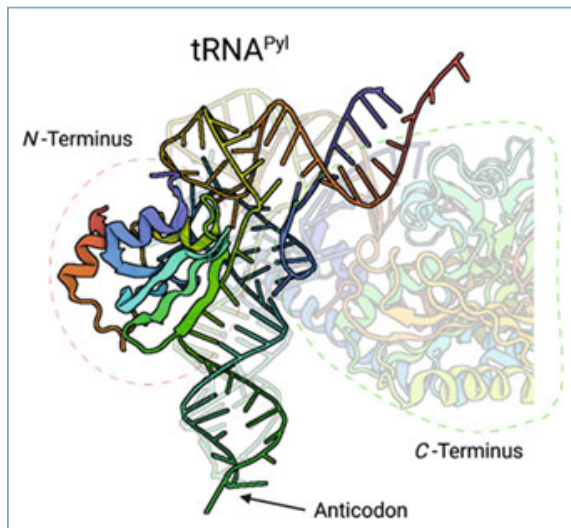

A Abb. 3: Erkennungsmechanismus der PyIRS von der tRNAPyl. Überlagerung der Kristallstruktur der N-terminalen Domäne von Methanosarcina mazei mit Bindung an die tRNA (PDB ID 5UD5) und der C-terminalen Domäne von Desulfitobacterium hafniense und dessen tRNA (PDB ID 2ZNI). $\mathrm{N}$ - und $\mathrm{C}$-Terminus der PyIRS erkennen die tRNA im Bereich des Anticodonarms. Dieser besitzt im Gegensatz zu kanonischen tRNAs nur einen winzigen variablen Arm, was die Orthogonalität zu allen anderen kanonischen tRNA/aaRS-Paaren erklärt. Auch ist zu erkennen, dass das Anticodon nicht am Erkennungsmechanismus beteiligt ist. Mit BioRender.com erstellt.
Methanosarcinales sind die einzigen Organismen, bei denen bekannt ist, dass sie auf allen drei Wegen Methan bilden können. Untersuchungen zeigten, dass zum Einbau von Pyrrolysin in die Proteinsequenz nur die spezifische tRNA (tRNA ${ }^{\text {Pyl }}$ ) und die dazugehörige Pyrrolysyl-tRNA-Synthetase (PylRS) notwendig ist (Abb. 3). Es handelt sich also um ein natürlich entstandenes Äquivalent zu den oben beschriebenen synthetisch gemachten OTS [11].

\section{Die erste Welle: Erste Nutzung des PyIRS-Systems für die Erweiterung des genetischen Codes}

Die PylRS ist ein Enzym, welches einen vergleichsweise unspezifischen Mechanismus zur Substraterkennung besitzt. Die Erkennung findet über eine tiefe Enzymtasche und bedingt unspezifischen hydrophoben Wechselwirkungen mit dem endogenen Substrat statt. Diese Eigenschaft wurde Anfang der 2000er-Jahre ausgenutzt, um mit dem nativen oder leicht mutierten PylRS-System eine Reihe von biochemisch/strukturbiologisch nützlichen Pyrrolysin-Derivaten in rekombinante Proteine einzubauen [12]. Obgleich so einige nicht kanonische Aminosäuren einge- baut wurden, die bioorthogonale funktionelle Gruppen aufwiesen (hauptsächlich Alkin-, Alken- und Azid-Gruppen), waren die Anwendungsgebiete doch deutlich eingeschränkt. Da es sich um Pyrrolysin-Analoga handelte, waren alle Substrate lang und voluminös. Ein Hauptproblem ist die hohe Flexibilität der langen Seitenkette. Sie verursacht z. B. Probleme in spektroskopischen Anwendungen bzw. limitiert den zu erhaltenden Informationsgehalt deutlich.

\section{Die zweite Welle: Engineerte PyIRS- Enzyme für den Einbau von Phenylalanin und dessen Derivate}

Kavran und Kollegen fiel die hohe strukturelle Ähnlichkeit zwischen der PylRS und der bakteriellen Synthetase für den Einbau von Phenylalanin (PheRS) auf [7]. Das war eine bemerkenswerte Leistung, da die Sequenzhomologie von PylRS und PheRS sehr gering ist. Daraufhin führten die Arbeiten von Liu und Kollegen dazu, dass das Spektrum der Aminosäuren, welche mit dem PylRS-System eingebaut werden konnten, deutlich erweitert wurde [12]. Es konnten nun Phenylalanin und dessen Derivate eingebaut werden. Das löste zum Teil das Problem der hohen

\section{Entdeckung des PyIRS-Systems}

Erste Welle:

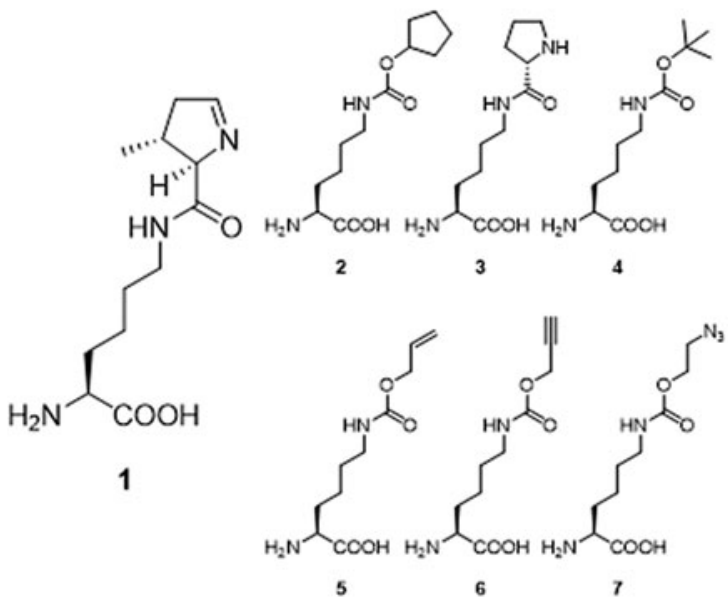

Zweite Welle:
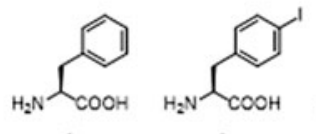<smiles>NC(Cc1ccc(Br)cc1)C(=O)O</smiles>

$$
\mathrm{H}
$$<smiles>COc1ccc(CC(N)C(=O)O)cc1</smiles><smiles>C1CCCCCC1</smiles><smiles>CCOc1ccc(CC(N)C(=O)O)cc1</smiles><smiles>C=C(/C=C\C)CC(C)N</smiles>

11

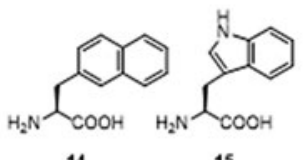

15

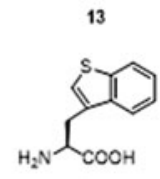

16
Dritte Welle:

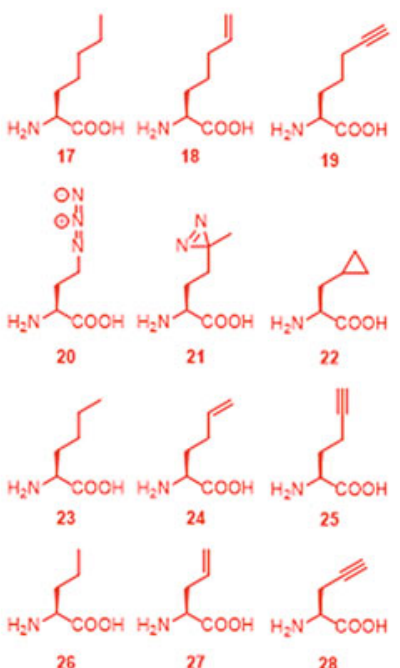

\section{Zeitstrahl}

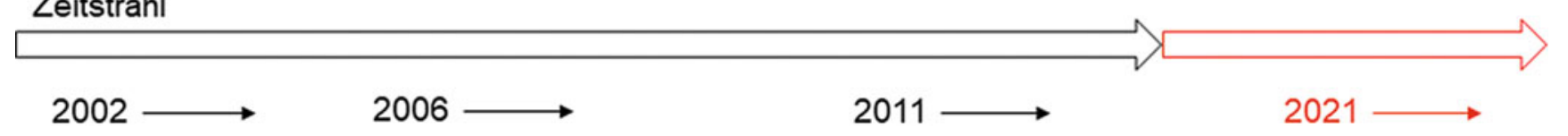

$\triangle$ Abb. 4: Zeitliche Abfolge der möglichen einzubauenden Substrate. Rot sind die in unserer Arbeitsgruppe neu eingebauten Substrate. Dies ist nur ein kleiner Bruchteil aller möglichen nicht kanonischen Aminosäurerepertoires, die mithilfe von PyIRS-basierten orthogonalen Paaren in rekombinante Proteine eingebaut werden können. Die außerordentliche Vielseitigkeit des natürlichen PylRS-Enzyms in Verbindung mit ausgereiften Methoden für die gerichtete Evolution von Enzymen (die auch die Verwendung von KI einschließen) hat eine solch dramatische Erweiterung des Anwendungsbereichs der ribosomalen Proteinbiosynthese ermöglicht. 
Flexibilität; auch konnte die Palette an funktionellen Gruppen noch erweitert werden (Cyano-Gruppen als spektroskopische Sonde). Diese Substrate waren allerdings immer noch sehr voluminös.

\section{Die dritte Welle: PyIRS-Enzyme für den Einbau von nicht aromatischen kleinen Substraten}

Die erste kleinere und nicht aromatische Aminosäure wurde von unserer Arbeitsgruppe in vivo eingebaut. Es handelte sich um ein Cystein-Derivat mit einer angehängten AllylGruppe (S-Allyl-L-cystein, Sac) [13]. Leider war die Effizienz, mit der diese Aminosäure eingebaut werden konnte, relativ schlecht. Weiterführende Arbeiten zur Verbesserung der Effizienz konnten zeigen, dass die Mutationen, die notwendig sind, damit die PylRS das Cystein-Derivat erkennt, zu einer deutlichen Destabilisierung des Enzyms führen. Somit lag das Enzym in einer unlöslichen und unfunktionalen Konformation im Proteinproduktionsorganismus (E. coli) vor. Wir konnten dieses Problem beheben, indem wir ein deutlich löslicheres Protein genetisch an das PylRS-Enzym fusionierten [14]. Mithilfe dieses Fusionsproteins wurden die Positionen aufgeklärt, die wichtig zur Erkennung von Sac sind. Außerdem bildete dieses Enzym dann die Grundlage für weitere Varianten, mit deren Hilfe erstmals kleine Aminosäuren (Abb. 4) mit einer Reihe von biochemisch nützlichen funktionellen Gruppen in Proteinsequenzen eingebaut werden konnten [15]. Unter anderem wird der Einbau dieser Substrate in verschiedene Proteine/Enzyme dabei helfen, ihre genauen Funktionsmechanismen spektroskopisch aufklären zu können.

Der Einbau dieser neuen Funktionen könnte dann auch mit der in vivo-nkAS-Synthese kombiniert werden. So könnten Zellen mithilfe des PyIRS-OTS dann in den einfachsten Nährmedien Proteine/Enzyme mit nicht natürlichen Funktionen/Eigenschaften herstellen. Zurzeit werden den Produktionskulturen von außen hauptsächlich chemisch synthetisierte Aminosäureanaloga zugeführt, was einen erhöhten Aufwand bedeutet. Daher ist die Kopplung der billigen und effizienten metabolischen Produktion von gewünschten nkAS mit OTS eine wichtige Richtung in der zukünftigen Forschung, die sowohl für den akademischen Bereich als auch für die biotechnologische Industrie wichtig ist [16].

\section{Literatur}

[1] Chin JW (2017) Expanding and reprogramming the genetic code. Nature 550: 53-60

[2] Furter R (1998) Expansion of the genetic code: Sitedirected p-fluoro-phenylalanine incorporation in Escherichia coli. Protein Sci 7: 419-426

[3] Wang L, Brock A, Herberich B, Schultz PG (2001) Expanding the genetic code of Escherichia coli. Science 292: 498-500

[4] Hao B, Gong W, Ferguson TK et al. (2002) A new UAGencoded residue in the structure of a methanogen methyltransferase. Science 296: 1462-1466

[5] Rothman DH, Fournier GP, French KL et al. (2014) Methanogenic burst in the end-permian carbon cycle. Proc Natl Acad Sci 111: 5462-5467

[6] Taubner R-S, Pappenreiter P, Zwicker J et al. (2018) Biological methane production under putative Enceladus-like conditions. Nat Commun 9: 748

[7] Kavran JM, Gundllapalli S, O’Donoghue P et al. (2007) Structure of pyrrolysyl-tRNA synthetase, an archaeal enzym for genetic code innovation. Proc Natl Acad Sci U S A 104: 11268-11273

[8] Kubyshkin V, Budisa N (2019) Anticipating alien cells with alternative genetic codes: away from the alanine world! Curr Opin Biotechnol 60: 242-249

[9] Kubyshkin V, Budisa N (2019) The alanine world model for the development of the amino acid repertoire in protein biosynthesis. Int J Mol Sci 20: 5507

[10] Enzmann F, Mayer F, Rother M, Holtmann D (2018) Methanogens: biochemical background and biotechnological applications. AMB Express 8: 1

[11] Namy O, Zhou Y, Gundllapalli S et al. (2007) Adding pyrrolysine to the Escherichia coli genetic code. FEBS Lett 581: 5282-5288
[12] Wan W, Tharp JM, Liu WR (2014) Pyrrolysyl-tRNA synthetase: an ordinary enzyme but an outstanding genetic code expansion tool. Biochim Biophys Acta - Proteins Proteomics 1844: $1059-1070$

[13] Exner MP, Kuenzl T, To TMT et al. (2017) Design of S-allylcysteine in situ production and incorporation based on a novel pyrrolysyl-tRNA synthetase variant. ChemBioChem 18: $85-90$

[14] Koch NG, Baumann T, Budisa N (2021) Efficient unnatural protein production by pyrrolysyl-tRNA synthetase with genetically fused solubility tags. Eingereicht.

[15] Koch NG, Goettig P, Rappsilber J, Budisa N (2021) Engineering pyrrolysyl-tRNA synthetase for the incorporation of non-canonical amino acids with smaller side chains. Eingereicht

[16] Völler J-S, Budisa N (2017) Coupling genetic code expansion and metabolic engineering for synthetic cells. Curr Opin Biotechnol 48:1-7

Funding note: Open Access funding enabled and organized by Projekt DEAL. Open Access: Dieser Artikel wird unter der Creative Commons Namensnennung 4.0 International Lizenz veröffentlicht, welche die Nutzung, Vervielfältigung, Bearbeitung, Verbreitung und Wiedergabe in jeglichem Medium und Forma erlaubt, sofern Sie den/die ursprünglichen Autor(en) und die Quelle ordnungsgemäß nennen, einen Link zur Creative Commons Lizenz beifügen und angeben, ob Änderungen vorgenommen wurden. Die in diesem Artikel enthaltenen Bilder und sonstiges Drittmaterial unterliegen ebenfalls der genannten Creative Commons Lizenz, sofern sich aus der Abbildungslegende

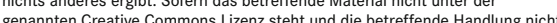
nach gesetzlichen Vorschriften erlaubt ist, ist für die oben aufgeführten Weiterverwendungen des Materials die Einwilligung des jeweiligen Rechteinhabers einzuholen. Weitere Details zur Lizenz entnehmen Sie bitte der Lizenzinformation auf http://creativecommons.org/licenses/by/4.0/deed.de.

\section{Korrespondenzadresse:}

Prof. Dr. Nediljko Budisa

Institut für Chemie

Fakultät II

Technische Universität Berlin

Müller-Breslau-Straße 10

D-10623 Berlin

nediljko.budisa@tu-berlin.de

nediljko.budisa@umanitoba.ca

\begin{tabular}{|c|c|}
\hline AUTOREN & \\
\hline 2 & $\begin{array}{l}\text { Nediljko (Ned) Budisa } \\
\text { Studium Biologie \& Chemie, molekulare Biologie und molekulare Biophysik. } 1997 \\
\text { Promotion. 1997-2000 Postdoc am Max-Planck-Institut für Biochemie, Martinsried. } \\
2001-2005 \text { Habilitation in Biochemie, TU München. Seit Mai } 2010 \text { W3-Professor } \\
\text { (Biokatalyse) an der TU Berlin. Seit } 2018 \text { Professor für Chemie und Inhaber des Tier } 1 \\
\text { Canada Research Chair (CRC) für chemische synthetische Biologie an der University } \\
\text { of Manitoba, Winnipeg, Kanada. }\end{array}$ \\
\hline
\end{tabular}

